\title{
Miniature Marciniak Setup for in-situ SEM Observation of Damage Micro-mechanisms
}

\author{
J.P.M. Hoefnagels ${ }^{1, a}$, C.C. Tasan ${ }^{1,2}$, E.C.A. Dekkers ${ }^{3}$, M.G.D. Geers ${ }^{1}$ \\ ${ }^{1}$ Eindhoven Univ. of Tech., Dept. of Mech. Engg., P.O.Box 513, 5600MB, Eindhoven, Netherlands. \\ ${ }^{2}$ Materials Innovation Institute (M2i), P.O.Box 5008, 2600GA, Delft, Netherlands. \\ ${ }^{3}$ Eindhoven Univ. of Tech., Joint Technical Department, P.O.Box 513, 5600MB, Eindhoven, Netherlands.
}

\section{Scientific relevance}

The industrial and scientific interest for ductile damage and fracture has significantly increased in the last decades due to the popularity of new advanced materials, such as high strength steels and nanostructured metals [1]. To allow for detailed investigation of micro-events such as the damage micro-mechanisms, the deformation needs to be studied in real time with in-situ scanning electron microscopy, allowing for digital image correlation (DIC) of the high resolution images for local strain mapping [2], see e.g. Figure 1. These studies, however, were limited to deformation under uniaxial tension due to the constraints of in-situ SEM testing.

At the same time, parallel research efforts have focused on elucidating the effect of the followed strain path on the ductile deformation micro-mechanisms, and its consequence for the resulting macroscopic forming and fracture limits in sheet metal [3]. Unfortunately, in these studies the damage micro-mechanisms were studied from post-mortem fracture analysis [4], due to the absence of a miniaturized testing setup capable of deforming sheet metal up to failure in alternative strain paths (e.g. plane strain or biaxial tension).
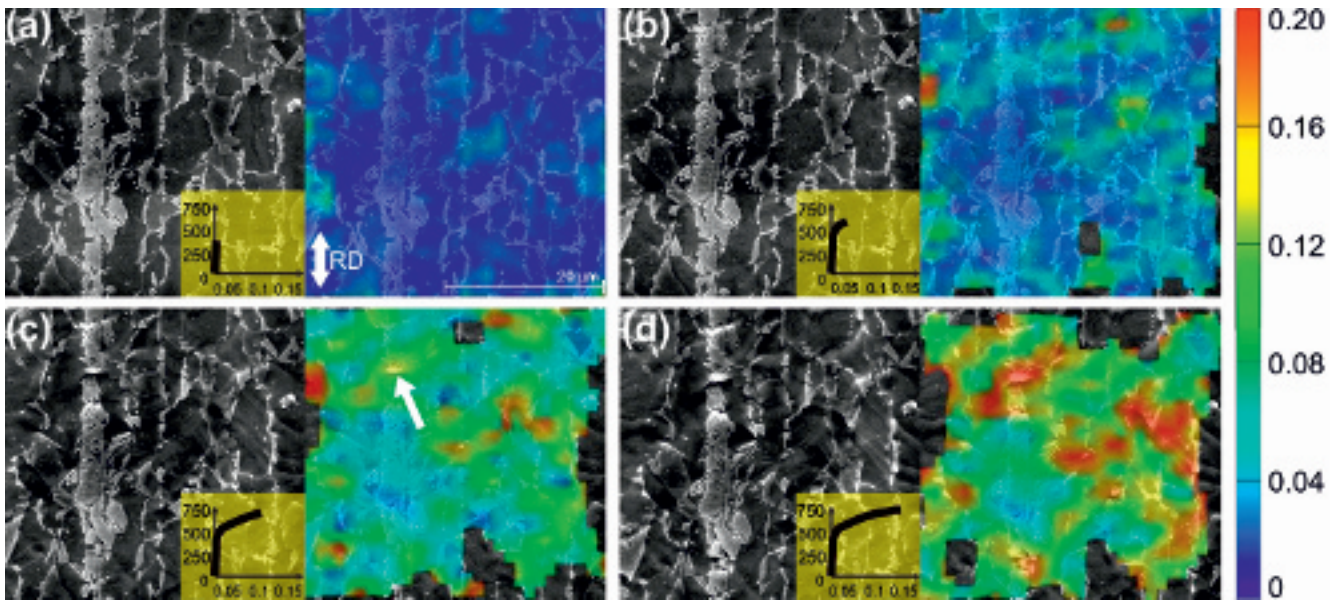

Fig. 1. SEM image and effective strain overlay at 4 different deformation stages for dual phase steel [7].

a e-mail: j.p.m.hoefnagels@tue.nl

This is an Open Access article distributed under the terms of the Creative Commons Attribution-Noncommercial License 3.0, which permits unrestricted use, distribution, and reproduction in any noncommercial medium, provided the original work is properly cited. 


\section{Research goal and results}

In this work, we set out to develop a test setup that allows for deformation of sheet metal at various strain paths up to the point of fracture under in-situ SEM observation. Figure 2(a) shows an experimental-numerical analysis of candidate testing setups (bulge test, Nakazima test, cruciform test, and Marciniak test), which identified the Marciniak test setup as the most suited for miniaturization [5]. Still, significant design challenges were identified, including (i) high load levels $(>100 \mathrm{kN})$ to be reached within the (small) SEM-chamber, (ii) forcing fracture to occur inside the "contactless" gauge section, and (iii) assuring the safety of the electron microscope.

\section{(a) Candidate tests}
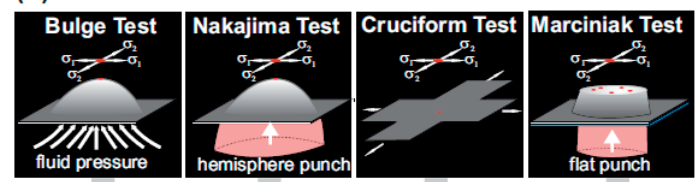

(b) Simulation Results
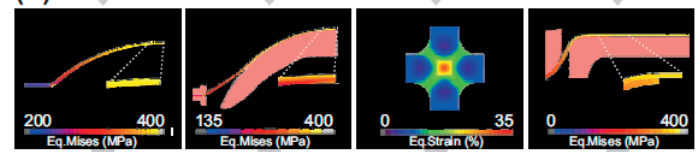

(c) Challenges in Miniaturization

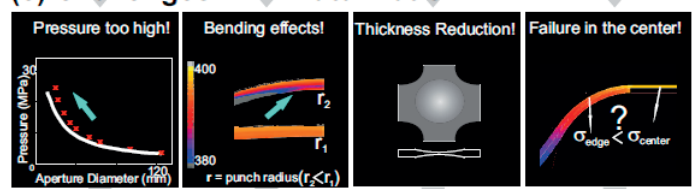

(a)

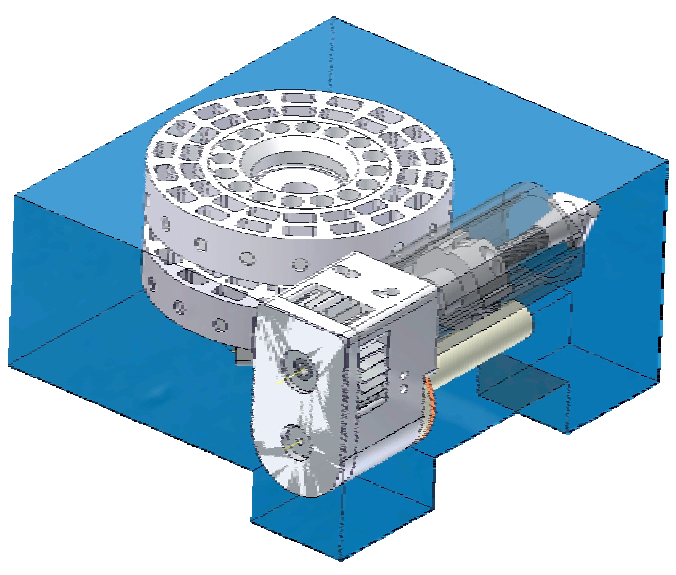

(b)

Fig. 2. (a) experimental/numerical evaluation of candidate multi-axial test setup for miniaturization, and (b) the final design of the miniaturized Marciniak Setup with the SEM vacuum chamber depicted in blue.

These challenges were successfully addressed and an in-situ miniaturized 150kN Marciniak setup (Figure 2(b)) was designed and built, which allows for world-first experimentation of multi-axial testing of sheet metal up to fracture under in-situ SEM observation. A case study is presented, in which strain-dependent damage initiation from microstructural bands in steels is investigated from SEM-DIC strain maps.

1. S. Sadagopan. et al., AISI/DOE Technology Roadmap Program, 2003; K.S. Kumar. et al., Acta Mater., 51 (2003) 5743.

2. J. Kang, et al., Scripta Mater., 56 (2007) 999; C.C. Tasan, J.P.M. Hoefnagels, M.G.D. Geers, submitted for publication.

3. T. Kuwabara. et al., Int. J. Plasticity, 23 (2007) 385.

4. C.C. Tasan, J.P.M. Hoefnagels, M.G.D. Geers, Scripta Mater., 61 (2009) 20; C.C. Tasan, J.P.M. Hoefnagels, C.H.L.J. Ten Horn, M.G.D. Geers, Mech. Mater., 41 (2009) 1264.

5. C.C. Tasan, J.P.M. Hoefnagels, M.G.D. Geers, Proceeding of the SEM XI conference (2008); G. Quaak, M.Sc. Thesis, Eindhoven University of Technology (2008). 